\title{
Use of segment arrays to evaluate the strength of angular induction
}

\author{
ERNEST GREENE \\ University of Southern California, Los Angeles, California \\ and \\ SABAH AL-QUADDOOMI \\ Kuwait University, Safat, Kuwait
}

\begin{abstract}
The contribution of local and global influence on angular induction was evaluated by varying the orientation of individual segments that were organized into an array. It was found that some of the misprojection of an oblique is determined by the orientation of the individual segments, and some by the overall configuration of the segments into two parallel bands. These results are integrated into a model that views angular induction as a lateral bias among orientation-sensitive neurons, with global influence differing from local influence only as a matter of scale.
\end{abstract}

Angular induction is a term for the process by which the presence of one or more lines can affect the perceived angle of another line or its projection-that is, its extension along a collinear path. It is used as a neutral term without implying any mechanism underlying the induction process. The process most often has been demonstrated using classic illusions of angle or direction, such as the Poggendorff. Much insight has been gained from the many studies of this illusion, with the work of Hotopf, Ollerearnshaw, and Brown (1974), Weintraub and Krantz (1971), and Wenderoth and Beh (1977) being especially significant.

It is generally accepted, for example, that the induction is greater when the parallel lines lie vertical or horizontal to the observer (Weintraub \& Krantz, 1971) and when the segment being judged for collinearity lies at a oblique orientation. Most investigators acknowledge that parallel induction lines are more effective than isolated ones, though it is not entirely clear why this is the case.

Greene $(1987,1988)$ has provided a new perspective on this matter by showing that induction magnitude is related to the angle at which each of the parallel components is placed; in other words, if the standard parallel configuration is violated, allowing the two lines to be oriented independently of one another. Under these conditions, the overall misprojection of an oblique line segmentfor simplicity, hereafter called an "oblique" -is substantially determined by the relative angle that is formed between the two lines that bound the projection space.

In addition, in a previous study (Greene \& AlQuaddoomi, 1989), we showed that complex waveforms

This research was supported in part by the Neuropsychology Foundation. Sabah Al-Quaddoomi was on a sabbatical leave at USC from Kuwait University for the 1987-88 academic year. Correspondence may be addressed to Ernest Greene, Department of Psychology, University of Southern California, Los Angeles, CA 90089. can be used instead of long, straight lines, and that they produce angular induction that can be as strong or stronger than the classic illusion effects. Although the data might be interpreted in various ways, we propose that local and global components of the waveforms (or an array of segments, as used in the present experiments) each contribute to the overall induction effect. This corresponds to the approach suggested by Tyler and Nakayama (1984) for explaining the Zöllner and Fraser illusions.

As illustrated in Figure 1, the global alignment of segments into two horizontal bands that span the page provides one influence on the judgment of collinearity - what may be called the "global" induction effect. This influence combines with a local effect, which is determined by the orientation of individual segments, thus amplifying or canceling the projection bias. In the present experiments, we manipulated the orientation of local segments in each horizontal array, as well as the number of segments in the array that are proximal to the oblique segment, in order to evaluate how these local and global factors affect the strength of induction.

\section{METHOD}

The experiments used an adaptation of the horizontal version of the Poggendorff illusion, wherein subjects were required to project an oblique line segment across the space between the parallels, selecting a point which was judged to be collinear to the oblique. In the course of each experiment, the oblique was presented in all four quadrants of the page, thus providing a counterbalanced design.

Visual stimulus materials were created using an Apple Macintosh computer with a laser printer and Adobe Illustrator software. Arrays of short segments were presented on the page as parallel bands, as illustrated in Figure 2. Each page also contained the oblique in one corner, positioned at $30^{\circ}$ to the horizontal so that its projection would pass through the center of the page. The oblique made contact with the center of one of the segments of the array; thus, the general configuration was similar to a standard one-diagonal Poggendorff figure. 

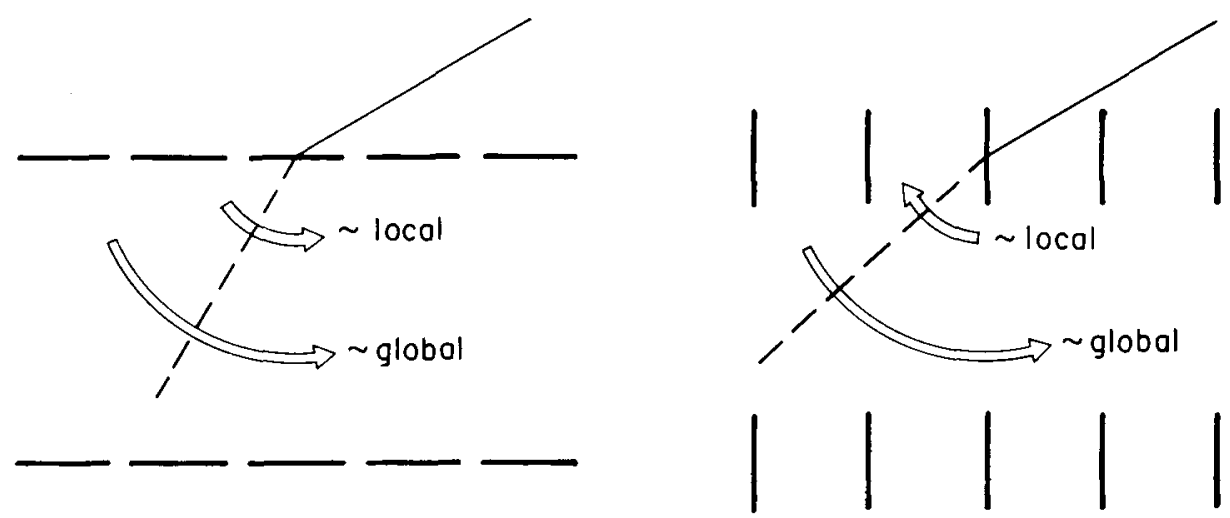

Figure 1. On the basis of prior results, it is hypothesized that the projection of an oblique line segment will be affected by the orientation of individual segments (the local effect) and by the overall alignment of the segments into parallel arrays (the global effect). Depending on the orientation of each component, the two influences may sum to produce greater misprojection, or may cancel to produce less. The arrows indicate the direction of induction on placement of the point judged to be collinear; their location does not imply the locus of action, or that the mechanism should be viewed as action by a force.

Three experiments were conducted using various configurations of the segment arrays. In each configuration, the individual segments were $7 \mathrm{~mm}$ long, and they were positioned with a horizontal separation of $8.4 \mathrm{~mm}$ at their centers. In the first two experiments, there were 29 segments in each of the parallel bands of the array, thus providing a $24.4-\mathrm{cm}$ span across all the elements. The arrays were separated by $6 \mathrm{~cm}$ (measured to the center of each band), and the oblique was $8 \mathrm{~cm}$ long. In addition, the stimulus sets for Experiments 1 and 2 included pages where simple horizontal-line parallels were used instead of the parallel arrays. This condition provided a control measure of the subjects' sensitivity to the induction effects.

The stimulus set for Experiment 1 varied the orientation of the segments in $15^{\circ}$ increments, rotating the segments in tandem about the center of each, and defining an angle relative to the oblique. The rotation convention is defined relative to the orientation of the oblique itself, which for this purpose was considered to have zero rotation irrespective of its true orientation on the page. To be comparable with previous studies, the angular convention for the segments was assigned on the basis of the axis of the segment bands, with rotation toward that axis being defined as positive, and away from that axis being defined as negative. Figure 3 illustrates this convention. For an oblique in the upper left quadrant of the page, clockwise rotation of the segment is defined as negative, and counterclockwise as positive. Since the two ends of the segment are perceptually equivalent, $180^{\circ}$ of rotation completes the cycle-that is, the $+90^{\circ}$ and $-90^{\circ}$ orientations are the same. The clockwisenegative, counterclockwise-positive convention also holds for an oblique that is presented in the lower right-hand corner of the page, but is reversed for obliques in the other two quadrants.

In Experiment 2, the orientation of segments in each of the parallel bands was manipulated independently. The two bands were designated as proximal and distal arrays on the basis of their proximity to the oblique, which is the source of the projection. One subset of pages provided stimuli in which the orientation of segments in the proximal array was varied in $15^{\circ}$ increments, while the distal array was held at $90^{\circ}$ relative to the oblique for all members of the group. The other subset held the segments in the proximal array to $90^{\circ}$, while the elements of the distal array were varied in $15^{\circ}$ increments.

Experiment 3 was designed to evaluate the relative contribution of the number of segments, where the orientation of these elements was held to one of four angles: $30^{\circ}, 45^{\circ},-30^{\circ}$, or $-45^{\circ}$. Each subset had $1,3,7,11$, or 15 segments, placed symmetrically on each side of the oblique as in the proximal arrays of Experiments 1 and 2 . In addition, stimulus pages were included that used a uniform horizontal line instead of segments, which corresponded to the collinear configuration of segments having a $30^{\circ}$ orientation. The length of the horizontal line varied across the subset of pages, matching the overall length of $3,7,11$, or 15 segments.

Therefore, the stimulus pages that comprised the set in each experiment were as follows. Experiment 1 used $15^{\circ}$ increments of segment orientation through $180^{\circ}$ of angle, plus a traditional uniform line configuration to provide a baseline measure of induction effect. Thus, there were 12 orientations of the segments and the uniform line control, repeated with the oblique being positioned in each of the quadrants $-13 \times 4=52$ configurations.

Experiment 2 used the same 12 variations of angle, manipulated separately for the proximal and distal arrays, as well as the longline control. These were repeated for each quadrant- $[(12 \times 2)+1]$ $\times 4=100$ configurations.

Experiment 3 used four orientations of segment alignment, plus the solid-line equivalent of the $30^{\circ}$ segment subset, each with five increments of length, with the addition of a bare oblique with no induction segment. Repeating across each quadrant, this provides $[(5 \times 5)+1] \times 4=104$ configurations.

Nine USC undergraduates with $20 / 20$ vision served as subjects for the study, a sampling of 5 subjects being tested in a given experiment. Thus, 4 of the subjects served in one experiment, 4 served in two, and 1 participated in each of the three experiments. For a given test session, each subject was provided with a stack of clean plain-paper copies of the stimulus pages in a random order and a viewing stand, following the methods of Weintraub and Brown (1986). The stand provided a viewing/marking surface inclined at $15^{\circ}$, with edge barriers to aid in the alignment of the stimulus pages. It also positioned a mask with eye slots held at $46 \mathrm{~cm}$ above the viewing surface. The horizontal placement of eye slots discouraged head turning, and thus promoted consistent alignment of the head and eyes as the stimulus pages were judged.

The subjects were given general instructions about the task requirements, but were not coached or shaped with respect to their judgments. For Experiments 1 and 2, the subjects were told that the goal was to determine how the bands of segments affected the accuracy of judging straightness. In particular, their task was to 

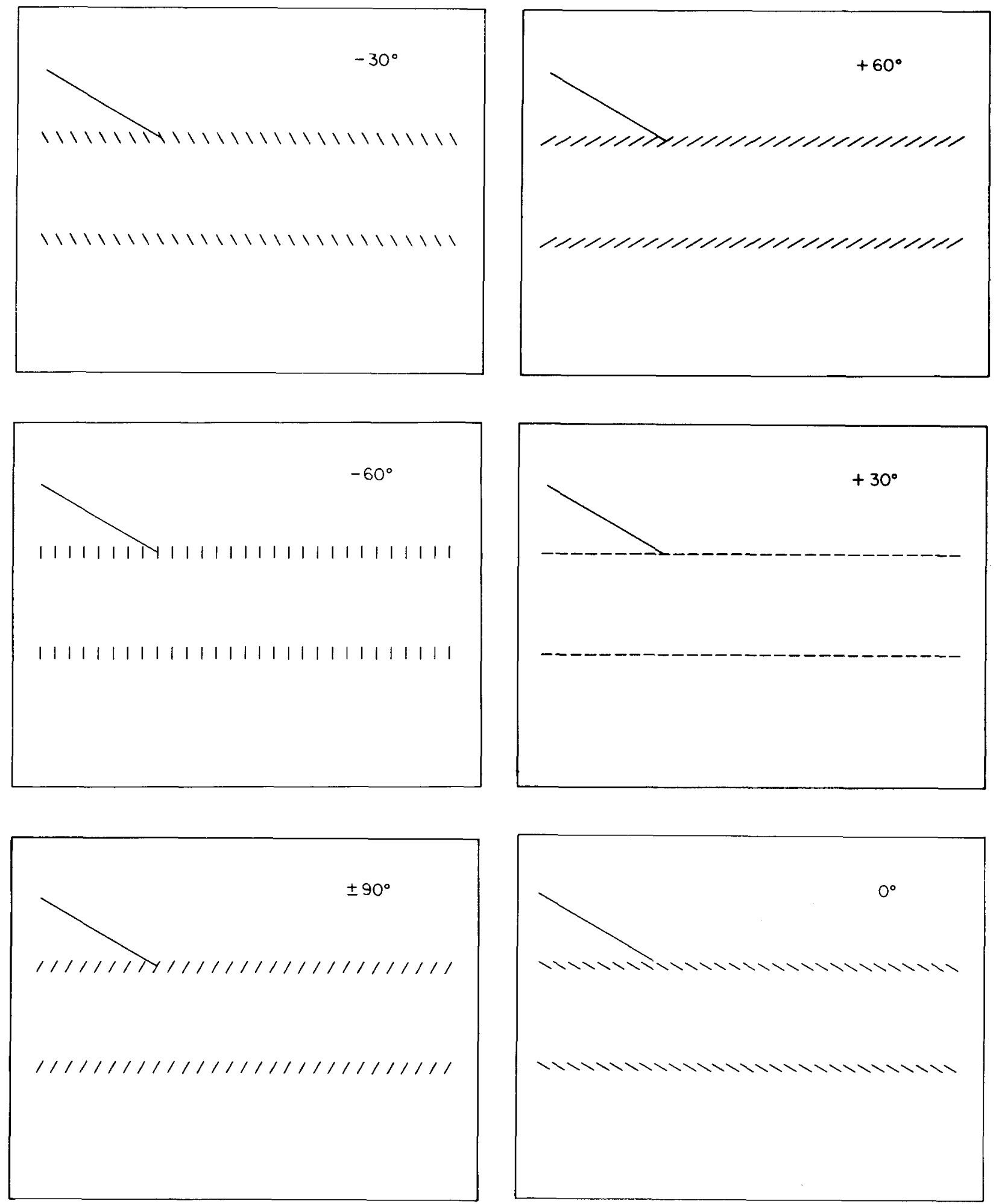

Figure 2. The induction stimuli consisted of horizontal arrays of short line segments, with the segments lying at various orientations relative to the oblique. The orientation of segments is labeled here, but these numbers did not appear on the stimulus pages judged by the subjects. 

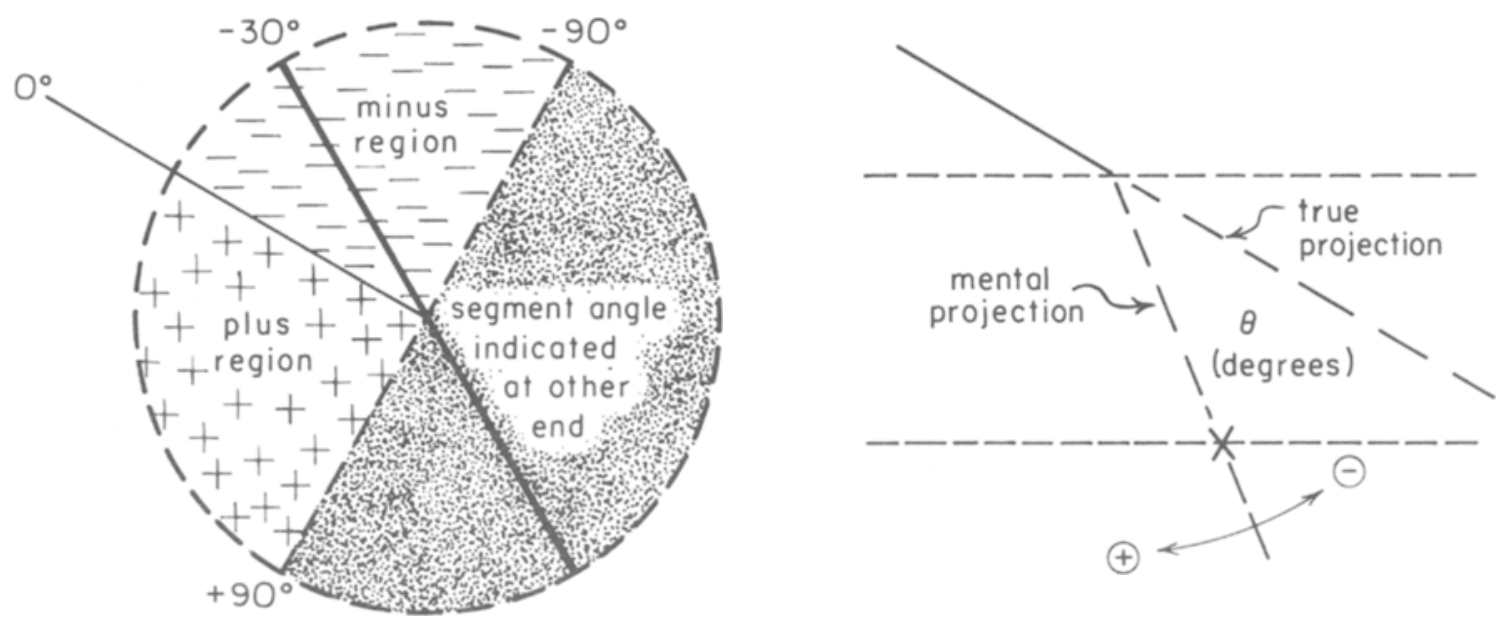

Figure 3. The convention for designating the orientation of segments is shown on the left. The angle is measured as a rotation about the center of the segment, relative to the medial tip of the oblique. Positive and negative angles are defined by clockwise and counterclockwise rotation of the segment, depending on the quadrant in which the oblique is located. The method for scoring misprojection is shown on the right. Note that the convention for defining the sign of the misprojection is opposite to what is used for the segment angle.

place a mark in the band of segments on the other side of the space from the oblique, at the point where the oblique would strike if it were drawn across the space. They were told not to draw the projection line, or to stroke the air as though the line were being drawn. For Experiment 3, each subject was told to place the mark "across the page" from the oblique, and by gesture a zone about $12 \mathrm{~cm}$ away from the tip of the oblique was indicated. Weintraub and Krantz (1971) have shown that induction effects are manifested as a constant angle-thus, one would not expect the projection distance to be a critical factor in any event.

Commonly, the instructions were repeated with variation of the phrasing, as well as gestures, until the experimenter was satisfied that the subject understood the requirements. Thereafter, the subjects were allowed to work at their own pace, and were encouraged to take breaks whenever they wished.

The subjects were paid for their participation, and were naive with respect to both the specific hypothesis and the phenomenon under investigation. For a given experiment, the stimulus set was judged twice, using a different random order of pages for each test session. These test sessions generally required $40-50 \mathrm{~min}$, and with rare exception a subject would be tested only once on any given day.

Responses were evaluated with the aid of a straightedge and protractor, defining misprojection as the angle (measured to the nearest $0.5^{\circ}$ ) at which the perceptual projection departed from the true projection of the oblique, as shown in Figure 3. For obliques in the upper left and lower right sides of the page, clockwise rotation was defined as positive, and counterclockwise as negative. The opposite convention applied to the other two quadrants. This standard was adopted to conform to the normal direction of induction effect where uniform lines are used-that is, producing positive misprojection with positive orientations of the induction line, and negative misprojection for negative orientations of the line.

\section{RESULTS}

\section{Experiment 1}

In Experiment 1, the orientation of segments was varied in $15^{\circ}$ increments to evaluate whether manipulation of this angle would affect the judgment of collinearity of the oblique. For each subject, the angle of misprojection across the four quadrants and two repetitions of the test were averaged, providing the raw data that were used in statistical analyses and data plots.

The question as to whether the orientation of segments affected projection of the oblique was tested using a oneway repeated measures analysis of variance (ANOVA). This analysis showed that segment angle was a significant factor in the degree of misprojection $[F(11,44)=$ $5.78, p<.001]$. The pattern of effects can be seen in Figure 4, which depicts the average of the 5 subjects as a function of this angle. By our convention, we begin the plot with responses to the $90^{\circ}$ induction angle (relative to the oblique), with the progression of negative through positive angles being shown from left to right. Note that the $90^{\circ}$ value is shown at both ends of the graph, identified as $-90^{\circ}$ on the left and $+90^{\circ}$ on the right. This convention allows completion of the cycle without having to physically wrap the ends of the chart.

The most striking aspect of these data is the fact that the positive angles (i.e., those that tilt toward the axis of the array) caused misprojection values to be consistently above the mean for all segments, and negative segment orientations (i.e., those that tilt away from the axis of the array) produced values that fell below the mean. This effect was confirmed by reanalyzing the data using a twoway repeated measures ANOVA, identifying the sign and tilt of the local segments as separate factors. Only the orientations having a sign were used for this analysisthat is, the positive and negative positions of $75^{\circ}, 60^{\circ}$, $45^{\circ}, 30^{\circ}$, and $15^{\circ}$, with the data at $\pm 90^{\circ}$ and $0^{\circ}$ being excluded. The results of this ANOVA showed that there were significant differences as a function of $\operatorname{sign}[F(1,4)=$ $101, p<.001]$, as well as significant effects for the sign $\times$ tilt interaction $[F(4,16)=4.5, p<.004]$. The tilt factor 
(A)

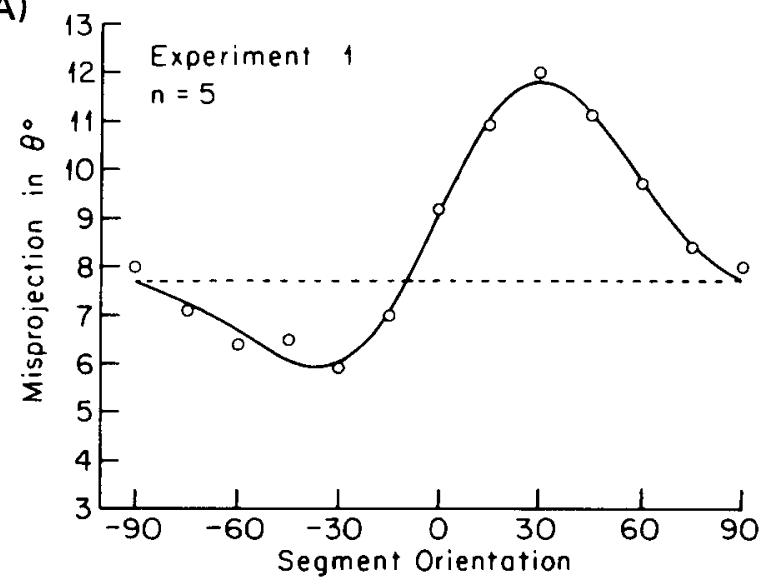

(B)
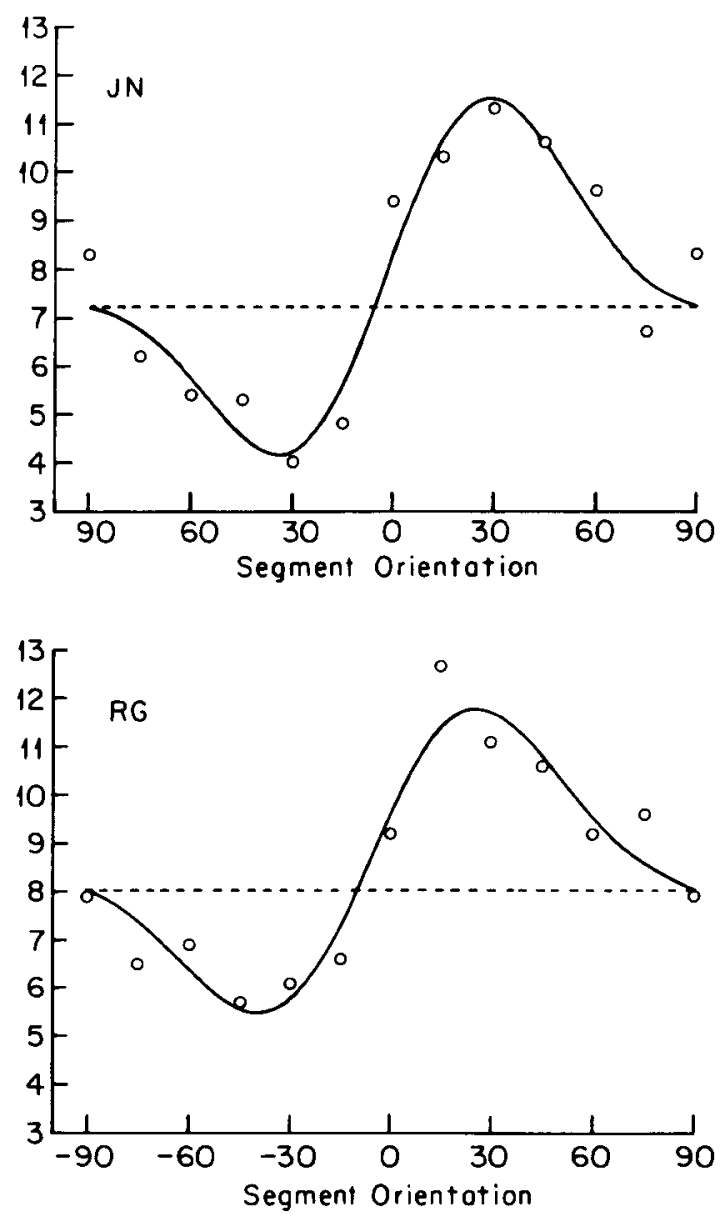

Figure 4. Mean misprojection of the 5 subjects (A) and scores of 2 individuals (B) at each segment orientation in Experiment 1. The data have been fitted using a second-order Fourier series. The dashed line shows the baseline of the curve. It is hypothesized that the baseline reflects a constant perceptual bias produced by the global alignment of the arrays, with positive segment angles acting to increase misprojection and negative angles acting to decrease it. was not significant, as would be expected given that each positive orientation was offset by a negative orientation.

We have fitted a line to the data using a Fourier series, breaking the period into $15^{\circ}$ increments, and retaining only two sine/cosine coefficients. This second-order Fourier curve is shown superimposed on the data points in Figure 4. As with the relative value of the points themselves, the maximal and minimal inflection peaks are found at $30^{\circ}$ on each side of the oblique orientation, and the baseline for the curve lies near the orthogonal positionthat is, at $90^{\circ}$. The $90^{\circ}$ orientation, therefore, might be viewed as a null point for the local effects.

The pattern of the effect is generally consistent with previous reports of line interaction. Bouma and Andriessen (1970) found positive and negative misprojection of an oblique line segment as a function of the orientation of the inducing line, and Carpenter and Blakemore (1973) reported similar effects using an orientation-matching task. In addition, Greene and Pavlov (1989) have shown that relatively long induction lines will produce (by our scoring convention) a positive deflection with positive angles and a negative deflection with negative angles; lines that lie at $90^{\circ}$ to the oblique do not cause much misprojection and are thus relatively neutral. The baseline seen in Figure 4 may reflect the contribution of the global alignment of the parallel arrays, which was a constant feature of the stimulus set. These parallels were positioned at $30^{\circ}$ to the oblique, producing a "global" perceptual bias-a constant $8^{\circ}$ of misprojection. To this effect, the sign and tilt of the local segments provided a supplementary or antagonistic influence, as reflected in Figure 4.

The effect of positive segment orientations appears to be stronger than negative orientations, and the source of this asymmetric influence is unknown. It should also be noted that the maximum induction produced in the array was approximately the same as the mean of the solid-line control figure $\left(12.0^{\circ}\right.$ and $11.6^{\circ}$, respectively). This indicates that the process of line segmentation does not, in itself, work to counteract the induction.

\section{Experiment 2}

For Experiment 2, the 12 orientations were again classified according to the sign and tilt of the segments, using only those that have a sign. Proximal versus distal location of the manipulated angle was the third factor; thus the data were analyzed using a three-way repeated measures design. As was true in the first experiment, tilt was not a significant influence due to the counterbalancing effect at each of the angles. The significant factors were the sign of the angle $[F(1,4)=46.9, p<.01]$, the proximal/distal location of the segments being manipulated $[F(1,4)=8.9, p<.05]$, the sign $\times$ tilt interaction $[F(4,16)=15.7, p<.0001]$, the sign $\times$ proximal $/$ distal interaction $[F(1,4)=9.7, p<.05]$, and the three-way interaction $[F(4,16)=3.5, p<.05]$.

The sources of the effects are made clear by inspection of the data plots shown in Figure 5. Varying the orienta- 

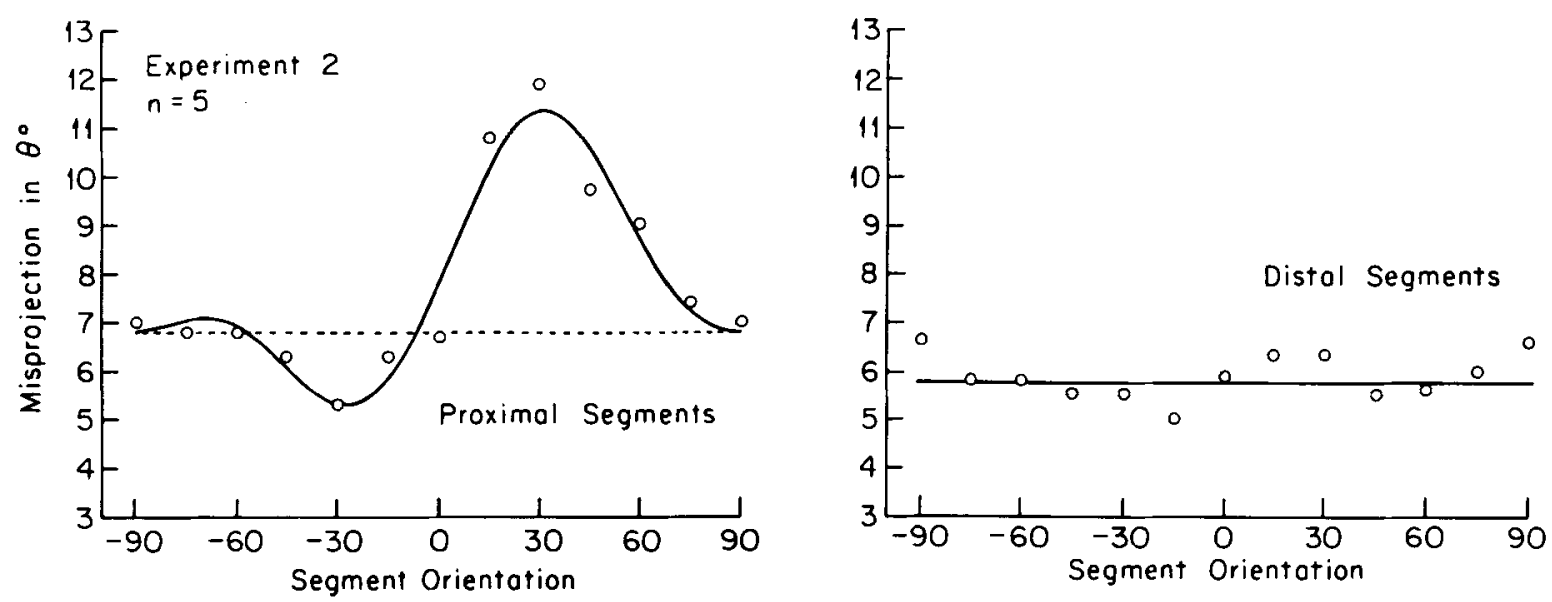

Figure 5. Varying the orientation of proximal segments produces the same general pattern of positive and negative effects. However, the orientation of distal segments is not a factor in the strength of induction influence.

tion of segments in the proximal array had a substantial effect on misprojection, and the best-fit curve for this data shows the pattern of negative/positive swings that was seen in Experiment 1. By contrast, varying the orientation of segments in the distal array did not produce a systematic effect on the size of the induction effect. On average, the misprojection produced by negative angles was within $0.5^{\circ}$ of that produced by positive angles, and the Fourier data fit did not suggest that the small variations followed the pattern produced by the proximal array. Thus, we have drawn a straight line across the graph at the level of the mean. The small variation of points might be due to chance, although in relation to this baseline there appears to be some hint of positive and negative deflection in the expected direction.

\section{Experiment 3}

The data for Experiment 3 were evaluated using a threeway repeated measures ANOVA, the factors being sign, tilt, and the number of segments in the array. Each of the factors provided a significant influence on projection error: the $F$ and $p$ values were $232.9,7.1,7.5$, and .001 , $.06, .01$, respectively. The tilt $\times$ segment number interaction and the three-way interaction were not significant, but the sign $\times$ tilt and sign $\times$ segment number interactions were, with $F$ values of 19.4 and 5.3, and $p$ values of .05 and .01 , respectively.

The substantial impact of the sign and orientation of segments can be seen by the separation of values in Figure 6: positive angles produced positive displacement of the oblique and negative angles caused negative misprojection. In addition, in keeping with the relative magnitude of influence seen in the first two experiments, the $30^{\circ}$ angles were more influential (in each respective direction) than the $45^{\circ}$ angles. There was a greater differential effect for positive than for negative segment orientations, which may relate to the greater overall induction effectiveness of positive angles, as reflected in Figures 4 and 5.

There is a general overlap of lines that connect the $30^{\circ}$ segment and $30^{\circ}$ solid-line points. The data suggest that a collinear configuration of segments is equivalent to a continuous line in terms of its effect in causing the oblique to be misprojected.

It appears that segments that are closest to the oblique make the strongest contribution to induction, with the effect being differential as a function of orientation. For positive angles, the induction grows to a maximum, or near maximum, in one to three segments. Thereafter, the addition of more segments does little to increase the misprojection influence, although some rise can be seen in the data of individual subjects. For negative angles, there is a strong negative swing, followed by a gradual climb toward less negative values. This pattern is consistent with the view that the orientation of the segments (which for negative angles would produce negative induction) is working at odds with the overall alignment of the segment array (which lies at a positive $30^{\circ}$ orientation). This hypothesis is discussed in greater detail below.

Finally, it should be noted that the peak effect in Experiment 3 is only half the maximum seen in the earlier experiments. It is conceivable that the magnitude of influence of the proximal array is limited to $6^{\circ}$, and that this is summed with the global effects that are produced by the additional presence of a second, parallel array. Greene and Pavlov (1989) provide data that suggests that each line in the conventional Poggendorff figure makes an independent contribution to the projection error, and the overall level of misprojection is derived (approximately) by the summation of their separate effects. The same may be true here, with the global influence from the two parallel bands together providing up to $8^{\circ}$ of projection bias (as reflected in the baseline of the Fourier curve). To this, the orientation of proximal segments 


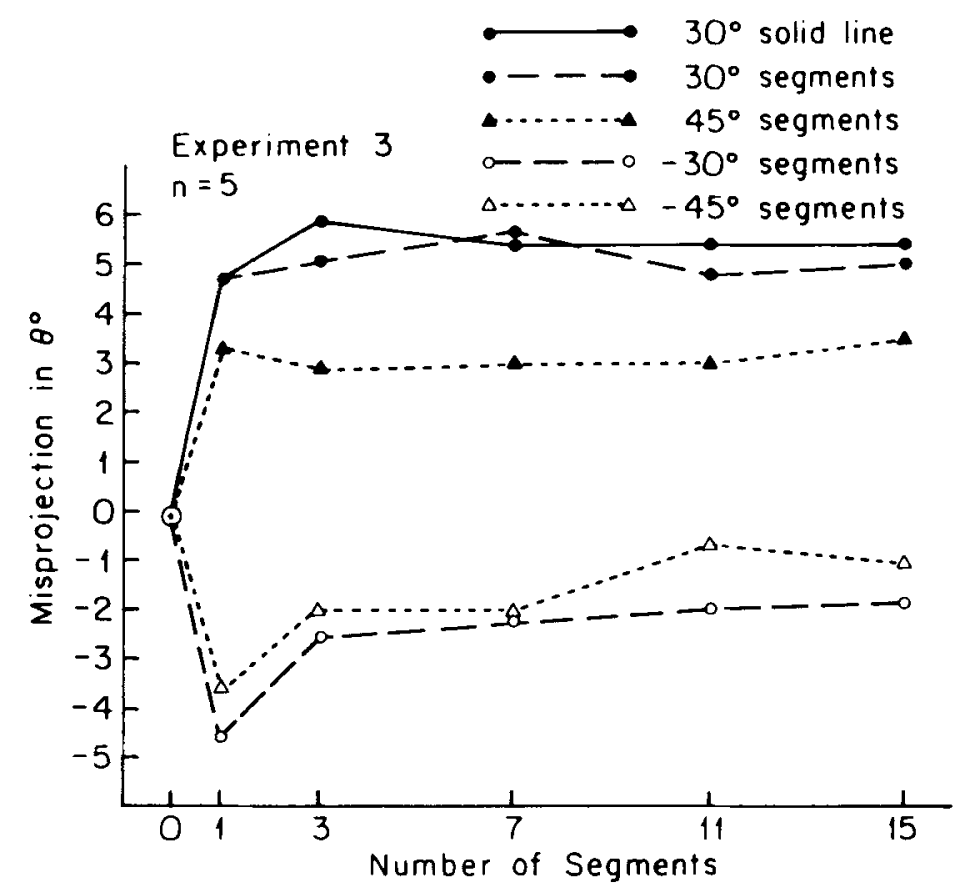

Figure 6. An additional indication of local versus global effects is provided by varying the number of segments in the proximal array. The local influence accounts for upwards of half the overall induction that is produced with full parallel arrays. This local effect reaches a maximum (or near maximum) within 1-3 segments.

can add several degrees of additional influence-perhaps $4^{\circ}-6^{\circ}$ of supplemental effect from the segments that lie directly adjacent to the tip of the oblique.

\section{DISCUSSION}

The results of these experiments suggest that angular induction effects in the Poggendorff illusion involve the interaction of local and global influences. This model builds on the views of Bouma and Andriessen (1970) and Carpenter and Blakemore (1973), who suggested that angular induction is produced by lateral inhibition among neurons that are sensitive to the orientation of stimulus lines. Further work has been done to separate local and global orientation factors in the Zöllner and Fraser illusions (Oyama, 1975; Tyler \& Nakayama, 1984).

In Experiments 1 and 2, subjects were asked to project an oblique through a zone that was bordered by parallel arrays of disconnected segments, the configuration being similar to a one-oblique Poggendorff figure. The orientations of segments in one or both arrays were varied in tandem, with the angle being designated as positive or negative relative to the orientation of the oblique (as shown in Figures 2 and 3). The pattern of results suggests that the parallel arrays produce a baseline of induction (about $8^{\circ}$ ), to which positive segment orientations can add, and from which negative segment orientations can subtract, influence. In other words, it appears that the broad sweep of the segments provides what may be described as a global influence, in that the relative position of the elements over a large extent of the visual field is a factor in the misprojection of the oblique. This is combined with the local influence, which is provided by the tilt of the individual segments.

Similar configurations of segment arrays have been used to study the Zöllner and Fraser illusions (Oyama, 1975; Tyler \& Nakayama, 1984). Oyama (1975) varied the orientation of segments that were positioned to form two parallel arrays, and found that the tilt of the individual segments affected the perceived orientation of the arrays, causing them to look as though they were not parallel. Tyler and Nakayama (1984) confirmed these effects, and further conducted a parametric study that showed a biphasic curve of influence with changes in the segment angle. Apparently, the perceived tilt of the segment array is biased in one direction when the tilt of the individual segments is within about $15^{\circ}$ of the actual tilt of the array, and it is biased in the other direction when the individual segments differ from the tilt of the entire array by $15^{\circ}-60^{\circ}$.

On the basis of these results, Tyler and Nakayama (1984) developed a quantitative model of the spread of orientation excitation and inhibition between receptive field responses to different line lengths. They also provided a useful illustration of how the local and global stimulus configurations may combine their influence by stimulating "channels" having small and large receptive fields, respectively. Figure 7 is an adaptation of their 

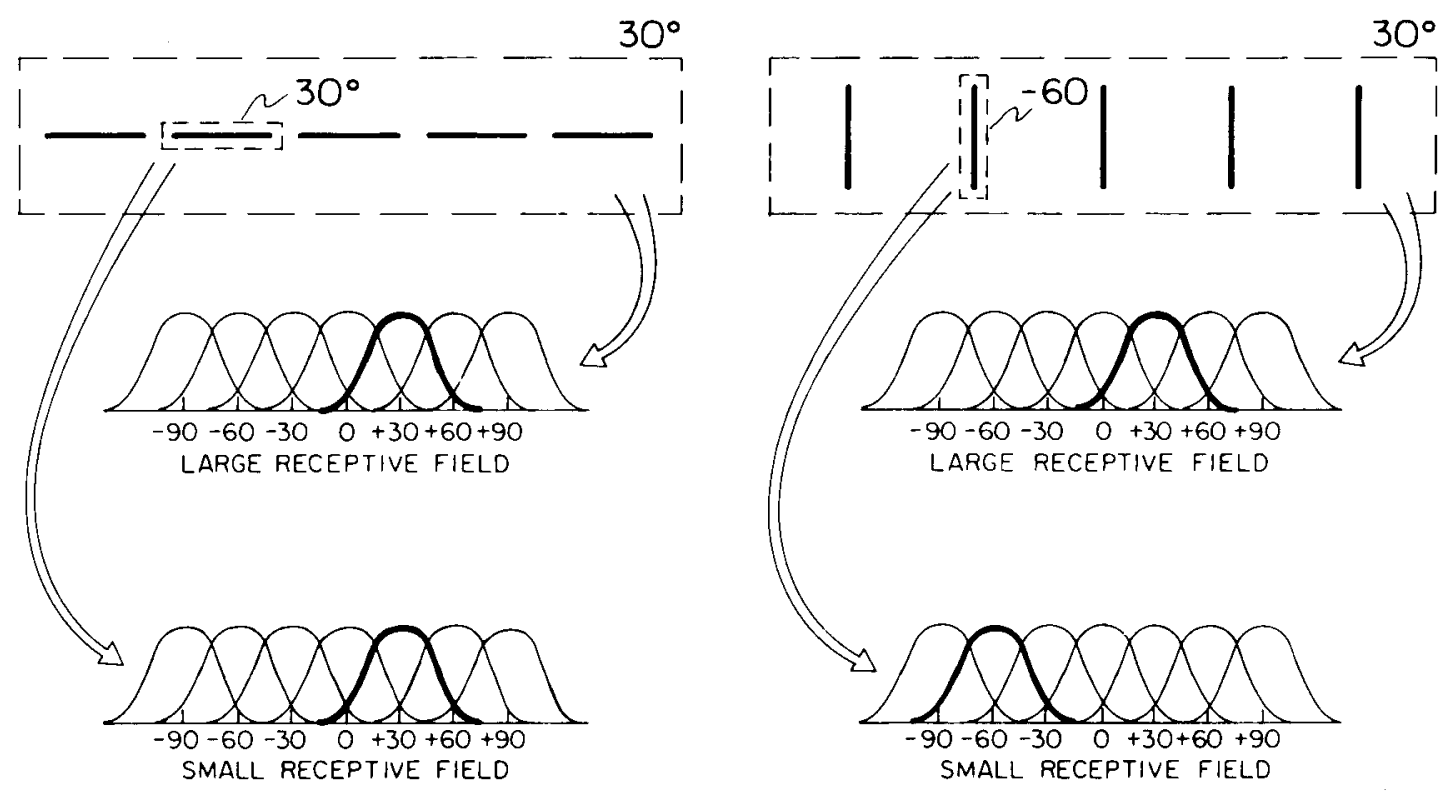

Figure 7. These results are interpreted according to the model of Tyler and Nakayama (1984), in which the orientation of segments, as well as the orientation of the full array, is registered respectively on small and large receptive fields. These receptive fields, in turn, control the activity of neurons, which mutually interact to bias perception of the angle of the oblique and/or its projection.

diagram, showing activation of orientation-sensitive channels (which might be specific cortical neurons) as a function of the tilt of the individual segment and of the overall alignment among segments. The responses of smalland large-scale channels combine to add or subtract their effects, presumably by means of excitation or inhibition among cortical neurons. In the present study, the adding of influence would produce larger misprojection of the oblique, whereas competing effects (from tilts having opposite signs) would reduce the degree of misprojection.

It should be noted that the interaction of local and global factors is somewhat simpler for the Poggendorff illusion than for the Zöllner and Fraser illusions. Here, the task calls for projection of an oblique, and the local and global effects either combine or cancel to produce greater or smaller degrees of misprojection. The task for the Zöllner and Fraser illusions is to judge the orientation of the arrays themselves, either by indicating when two arrays are parallel or by setting a single array to match a major axis of the observer. With these tasks, the direction of effects can go either way, in that the local tilt can bias the judgment of global alignment, or vice versa. Indeed, biphasic influence was exactly what Tyler and Nakayama (1984) found, and they demonstrated that one direction corresponded to the Fraser illusion and the other to the Zöllner.

There were no clear indications of Fraser/Zöllner effects with the stimuli used in the present experiments. In Experiment 1, the tilt of the individual segments of the two arrays was in the same direction, and any local-to- global bias would be to turn them alike-that is, keeping them parallel. Although we cannot discern any shift of the horizontal alignment of the arrays, careful experimental study might show such an effect. On the other hand, one would expect such local/global interactions to produce a conspicuous shift from parallel alignment when the local segments in the two arrays were different, and yet under these conditions, the arrays still looked parallel. We might speculate that the large separation between the two arrays, the use of relatively short segments, and the large span all contributed to the stability in the perceived tilt of the global arrays.

In two of the three experiments, there were two parallel bands of segments that had an axial alignment of $+30^{\circ}$ relative to the oblique. The separation of the bands, the overall length of the arrays, and other such factors were held constant to provide a backdrop against which the manipulation of local orientation could operate. This puts the major focus on the effects of segment angle. However, we think it would be a mistake to ignore the global contribution from each of two arrays-the one that is proximal to the oblique and the one that lies at a distal location. Using long-line stimuli, Greene $(1987,1988)$ has shown that proximal and distal lines both contribute to the induction process. Furthermore, Greene and Pavlov (1989) showed that the influence of each line is determined by the angle it forms relative to the oblique, with misprojection of the oblique being approximately the sum of the independent induction effects. Although we have not 
manipulated the orientation of the global arrays in the present experiments, on the basis of the previous work, we think it likely that both the proximal and the distal arrays make a global contribution to the misprojection. In fact, as shown in Figure 6, where the distal array was not provided, the overall induction was only half the effect produced by the combination of two arrays in parallel.

The location of induction peaks at $+30^{\circ}$ and $-30^{\circ}$ deserves additional comment. The positive inflection peak is produced by a collinear alignment of the segments, thus being very similar to the configuration of long-line elements in the conventional Poggendorff figure. Collinearity itself is not essential for generating strong induction; for example, Greene and Al-Quaddoomi (1989) were able to produce greater than normal errors using a triangular wave pattern. Nonetheless, collinearity of segments may add somewhat to their effectiveness, and if this is the case, it could explain why the positive deflections of the Fourier curve were larger than the negative deflections.

In addition, it might seem that the alignment of segments along a cardinal axis is a contributing factor. It has often been asserted that the strength of this effect is greatest with horizontal or vertical alignment of the parallels; Weintraub and Krantz (1971) provided the most convincing experimental confirmation of this view. However, results from our laboratory suggest that projection error is determined primarily by the orientation of the induction line relative to the oblique, and for some stimulus configurations and test conditions, the maximal effects are produced at angles of $15^{\circ}$ or less-at orientations that do not correspond to alignment to the cardinal axes (Greene \& Pavlov, 1989, and results not yet reported).

In Experiment 2, we examined the relative contribution of segment orientation when it was varied separately in the proximal and distal arrays. Manipulating the orientation of proximal segments produced a clear pattern influence, as is clear from inspection of Figure 5. The local induction effects followed the pattern seen in Experiment 1 , being stronger for positive angles and weaker for negative angles. However, when the segments of this proximal array were held at $90^{\circ}$ and the orientation of distal segments was varied, there was only a slight indication that the manipulation produced differential effects.

Whatever might be the contribution of the distal array to global factors, we have minimal evidence that the specific orientation of its segments contributes to the induction process. Indeed, considering the results of Experiment 3 as well, it appears that it is the orientation of the 1-3 segments closest to the tip of the oblique that constitutes the local factor. Where these local segments have a positive angle relative to the oblique, they add to the misprojection, and where they have a negative angle they subtract from the overall effect. Thus, where there are only 1-3 segments having a negative orientation, one sees the sharp negative deflection as reflected in Figure 6. As one adds more segments, the strength of the local angle appears to be mitigated, presumably by the growth of global influence as the horizontal dimension of the band is expanded.

We have no preconception as to the neural locus of the induction process. Nevertheless, orientation sensitivity has been most extensively studied in the primary visual cortex, following the classic studies of Hubel and Wiesel (e.g., 1962, 1977), and biological models most often draw upon the observations at this site. In general, the process is modeled as the responses of, and interactions among, the neurons that map the various orientations of the stimulus line(s).

The individual neurons have a rather restricted "view" of the image, and thus one major issue is how the information can be combined for the perception of long lines. It has been suggested that long horizontal fibers within the cortex might connect cells having similar orientation tuning (Gilbert \& Wiesel, 1985; Mitchison \& Crick, 1982; Wiesel \& Gilbert, 1983), providing an extended lattice of circuitry through which the successive portions of a line can be integrated (Morgan \& Hotopf, 1989). Although this is an intriguing possibility, such a model faces a number of problems, not the least of which is anisotropylimits on the ability to combine information that does not fall along the axes of the lattice.

In addition, caution is advised in extending neurophysiological findings as models for human judgment. Although there is physiological and anatomical evidence for a lattice that connects cells having a similar orientation preference (Gilbert \& Wiesel, 1989; Ts'o, Gilbert, \& Wiesel, 1986), there is little evidence that it is designed to detect collinearity of line segments. A number of studies have shown that the neurons in the primary visual cortex respond vigorously to a grid of lines, and the data showing interaction of receptive fields could be drawn just as well toward the analysis of spatial frequency (or texture).

There are problems, as well, in fitting the dimensions of receptive fields and intrinsic connections to the scale of perceptual integration. Dow, Bauer, Snyder, and Vautin (1984) reported average receptive field size in primates to be $15-20 \mathrm{~min}^{2}$ in the vicinity of the fovea. Although there is some scatter of size, few receptive fields fall above $30 \mathrm{~min}^{2}$-less than $1^{\circ}$ of visual angle along the long axis. The segments in the stimulus material used in the present experiment were approximately $1^{\circ}$ in length, so any combining of effect at the global level would require an extended lattice of connections.

In primates, Rockland and Lund (1983) have observed intrinsic fibers of up to $4 \mathrm{~mm}$ in length. This may not be sufficient to provide a communication lattice between hypercolumn modules in the vicinity of the fovea, since Tootell, Switkes, Silverman, and Hamilton (1988) found the cortical magnification factor to be approximately $15 \mathrm{~mm}$ of tissue per degree of visual field. Furthermore, even if the Rockland and Lund data are too conservative, the pattern of results in the literature suggests that the function of the intrinsic lattice is to coordinate the activities of neighboring modules. In primates, there is minimal evidence that such a lattice could combine responses across 
an extended portion of the visual field, as would be needed to generate the global effects of the present experiments. We think it likely, therefore, that the summary of global alignment (as well as the perception of collinearity across such spans) is accomplished by the cascade of information into other brain areas-perhaps secondary visual cortex.

Although the present data will not support an extended discussion of induction mechanism, there is one final point that should be mentioned. A receptive-field/lateralinhibition model logically relates to the judgment of tilt and predicts that the inducing line will alter the perceived orientation of the oblique. The collinearity judgment requires that a point be placed across the space between the parallel arrays, and any error can be attributed either to the misperceived angle of the oblique being projected, or to a disruption of the projection process. The experimental conditions do not allow for separation of these alternatives. However, it may be noted that Carpenter and Blakemore (1973) evaluated the effect of an induction line on perceived orientation using a direct-matching paradigm, and reported maximal displacement of $2^{\circ}-3^{\circ}$. The overall misprojection error seen here is many times larger, which suggests that the induction effect cannot be completely explained as the misperception of tilt. An alternative concept, such as distortion of the spatial field, may be appropriate.

\section{REFERENCES}

Bouma, H., \& ANDriessen, J. J. (1970). Induced changes in the perceived orientation of line segments. Vision Research, 10, 333-347.

CARPENTER, R. H. S., \& BLAKEMORE, C. (1973). Interactions between orientations in human vision. Experimental Brain Research, 16, 287-303.

Dow, B. M., Bauer, R., Snyder, A. Z., \& Vautin, R. G. (1984). Receptive fields and orientation shifts in the foveal striate cortex of the awake macaque monkey. In G. M. Edelman, W. E. Gall, \& W. M. Cowan (Eds.), Dynamic aspects of neocortical function (pp. 41-65). New York: Wiley.

GILBERT, C. D., \& WiESEL, T. N. (1985). Intrinsic connectivity and receptive field properties in visual cortex. Vision Research, 25, 365-374.

Gilbert, C. D., \& Wiesel, T. N. (1989). Columnar specificity of intrinsic horizontal and corticocortical connections in cat visual cortex. Journal of Neuroscience, 9, 2432-2442.

GrEeNE, E. (1987). The relative contribution of contact and target lines in the magnitude of the Poggendorff effect. Perception, 16, 385-388.
Greene, E. (1988). The comer Poggendorff. Perception, 17, 65-70.

Greene, E., \& Al-Quaddoomi, S. (1989). Assessing the strength of angular induction using waveforms. Bulletin of the Psychonomic Society, 27, 245-248.

Greene, E., Pavlov, G. (1989). Angular induction as a function of contact and target orientation. Perception, 18, 143-154.

Hotopf, W. H. N., Ollerearnshaw, C., \& Brown, S. (1974). The regression to right angles tendency and the Poggendorff illusion: III. British Journal of Psychology, 65, 213-231.

Hubel, D. H., \& Wiesel, T. N. (1962). Receptive fields, binocular interaction, and functional architecture in the cat's visual cortex. Journal of Psysiology, 160, 106-154.

Hubel, D. H., \& Wiesel, T. N. (1977). Functional architecture of macaque monkey visual cortex. Proceedings of the Royal Society, London, Series B, 198, 1-59.

Mitchison, G., \& Crick, F. (1982). Long axons within the striate cortex: Their distribution, orientation, and patterns of connection. Proceedings of the National Academy of Sciences, 79, 3661-3665.

Morgan, M. J., \& Hotopf, W. H. N. (1989). Perceived diagonals in grids and lattices. Vision Research, 29, 1005-1015.

Oyama, T. (1975). Determinants of the Zollner illusion. Psychological Research, 37, 261-280.

ROCKLAND, K. S., \& LUND, J. S. (1983). Intrinsic laminar lattice connections in primate visual cortex. Joumal of Comparative Neurology, 216, 303-318.

Tootell, R. B. H., Switkes, E., Silverman, M. S., \& Hamilon, S. L. (1988). Functional anatomy of macaque striate cortex: 2. Retinotopic organization. Journal of Neuroscience, 8, 1531-1568.

Ts'o, D. Y., GilberT, C. D., \& Wiesel, T. N. (1986). Relationships between horizontal interactions and functional architecture in cat striate cortex as revealed by cross-correlation analysis. Journal of Neuroscience, 6, 1160-1170.

Tyler, C. W., \& Nakayama, K. (1984). Size interactions in the perception of orientation. In L. Spillmann \& B. R. Wooten (Eds.), Sensory experience, adaptation, and perception (pp. 529-546). Hillsdale, NJ: Erlbaum.

Weintraub, D. J., \& Brown, S. (1986). Perceptual plane geometry: Collinearity judgments probe the perceived orientation of an angle's sides. Journal of Experimental Psychology: Human Perception \& Performance, 12, 434-444.

Weintraub, D. J., \& Krantz, D. H. (1971). The Poggendorff illusion: Amputations, rotations, and other perturbations. Perception \& Psychophysics, 10, 257-264.

Wenderoth, P., \& BeH, H. (1977). Component analysis of orientation illusions. Perception, 5, 57-75.

WIESEL, T. N., \& GILBERT, C. D. (1983). Morphological basis of visual cortical function. Quarterly Joumal of Experimental Physiology, 68D, 525-543.

(Manuscript received December 12, 1988; revision accepted for publication September 18, 1989.) 